Chapter 16

\title{
Single living cell processing in water medium using focused femtosecond laser-induced shockwave and cavitation bubble
}

\author{
Y. Hosokawa ${ }^{\mathrm{a}, \mathrm{e}}$, R. Yasukuni ${ }^{\mathrm{a}}$, J.-A. Spitz ${ }^{\mathrm{a}, \mathrm{c}, \mathrm{d}}$, T. Tada ${ }^{\mathrm{a}}$, T. Negishi ${ }^{\mathrm{a}}$, \\ C. Shukunami ${ }^{\mathrm{b}, \mathrm{e}}$, Y. Hiraki ${ }^{\mathrm{b}, \mathrm{e}}$, T. Asahi ${ }^{\mathrm{a}}$, R. Méallet-Renault ${ }^{\mathrm{c}}$ and \\ H. Masuhara ${ }^{\mathrm{a}, \mathrm{e}}$
}

${ }^{a}$ Department of Applied Physics and Venture Business Laboratory, Osaka University, 2-1 Yamadaoka, Suita, Osaka, 565-0871, Japan

${ }^{b}$ Department of Cell Differentiation, Institute for Frontier Medical Sciences Kyoto University, 53 Kawahara-cho Shogoin, Sakyo-ku, Kyoto, 606-8507, Japan

${ }^{c}$ Laboratoire de Photophysique et Photochimie Supramoléculaires et Macromoléculaires (UMR 8531), Ecole Normale Supérieure de Cachan, 61, avenue du Pdt Wilson, 94235 Cachan Cedex, France

${ }^{\mathrm{d}}$ Laboratoire de Biotechnologies et Pharmacologie génétique Appliquée (UMR 8113), Ecole Normale Supérieure de Cachan, 61, avenue du Pdt Wilson, 94235 Cachan Cedex, France

${ }^{\mathrm{e}}$ CREST-JST

\section{INTRODUCTION}

When a near-infrared femtosecond laser is focused by a high-numericalobjective lens, the cell and tissue are three-dimensionally processed at the laser focal point through multiphoton absorption. Such phenomenom is possible due the transparency of cells and tissues in the near-infrared region. Recently, the 3D processing of biological material has attracted much attention as a new method for cell and tissue dissection with fine spatial selectivity [1-7]. In addition, the femtosecond laser processing has an advantage for biological materials which are sensitive to thermal damage. Indeedthe femtosecond laser processing in 
water (which is main component of biomaterials) is normally dived not only by thermal evaporation but also by shockwave and cavitation bubble generation. Although the latter generation can be induced also by near-infrared nanosecond and picosecond lasers [8], an efficient multi-photon absorption by femtosecond laser initiates these generations with relatively low pulse energy $[9,10]$. The shockwave and cavitation bubble generated are localized at the laser focal point and produce local processing of cell and tissue with less thermal damage. In fact, our group succeeded in the manipulation of single animal cell without thermal damage by applying the compact shockwave and cavitation bubble [11]. In this chapter, we explain the effective generation process of shockwave and cavitation bubble on the basis of photomechanical ablation process, and describe femtosecond laser processing for living animal cell.

\section{SAMPLE AND MICROSCOPE}

As a representative animal cell, NIH $3 \mathrm{~T} 3$ fibroblast was prepared. The fibroblasts were plated onto glass-bottom Petri dishes which had been coated by fibronectin $(5 \mathrm{mg} / \mathrm{ml})$ and cultured in Dulbecco's Modification of Eagle's Medium supplemented with $5 \%$ fetal bovine serum. The specimen was set on an inverted microscope (Olympus, IX71), to which a regeneratively amplified femtosecond Ti:Sapphire laser (Spectra-Physics, Hurricane, $800 \mathrm{~nm}, 120 \mathrm{fs}$ ) was led as shown in Fig. 1. The laser pulse was focused by a microscope objective lens. Single shot laser pulses were picked up from the pulse train with a repetition rate of $125 \mathrm{~Hz}$ by a mechanical shutter. Laser power was tuned by a half-wavelength plate and dual polarizers.

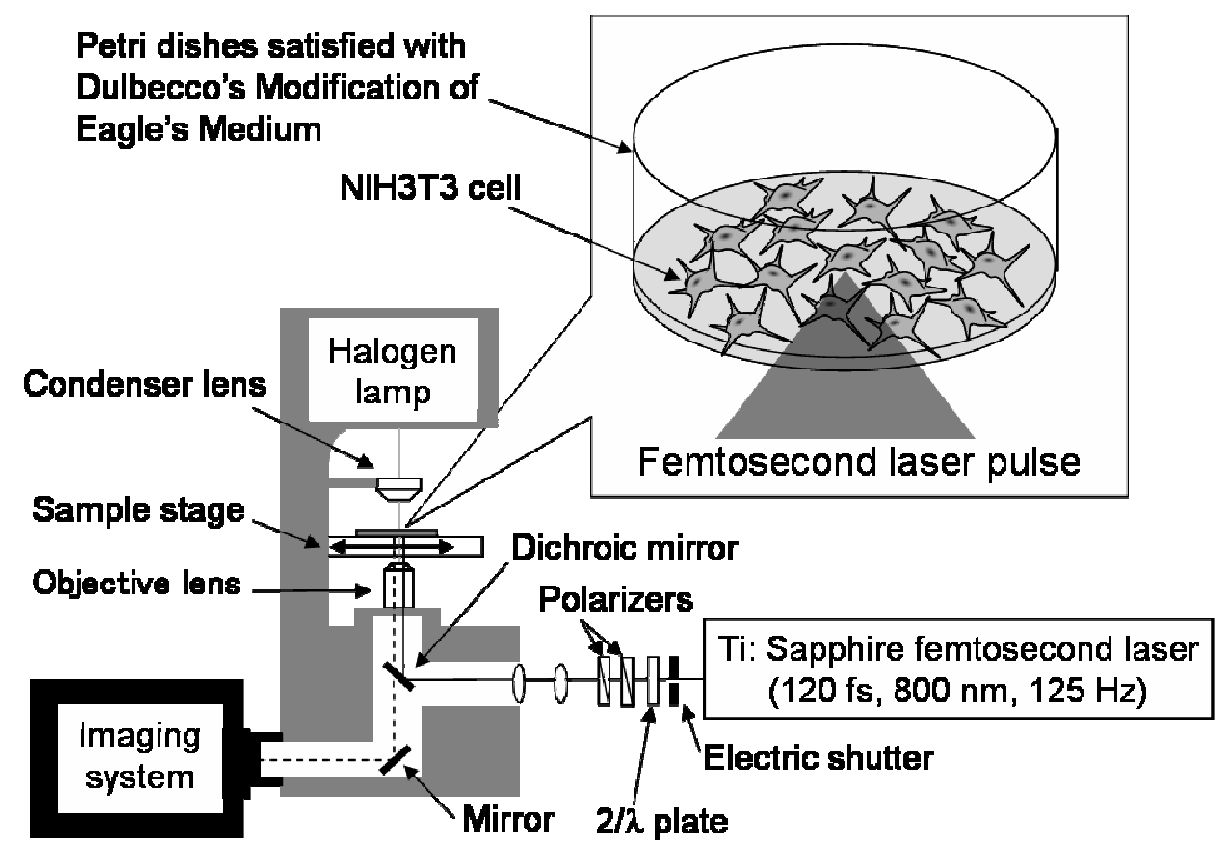

Fig. 1. Experimental setup of femtosecond laser processing for living animal cell. 


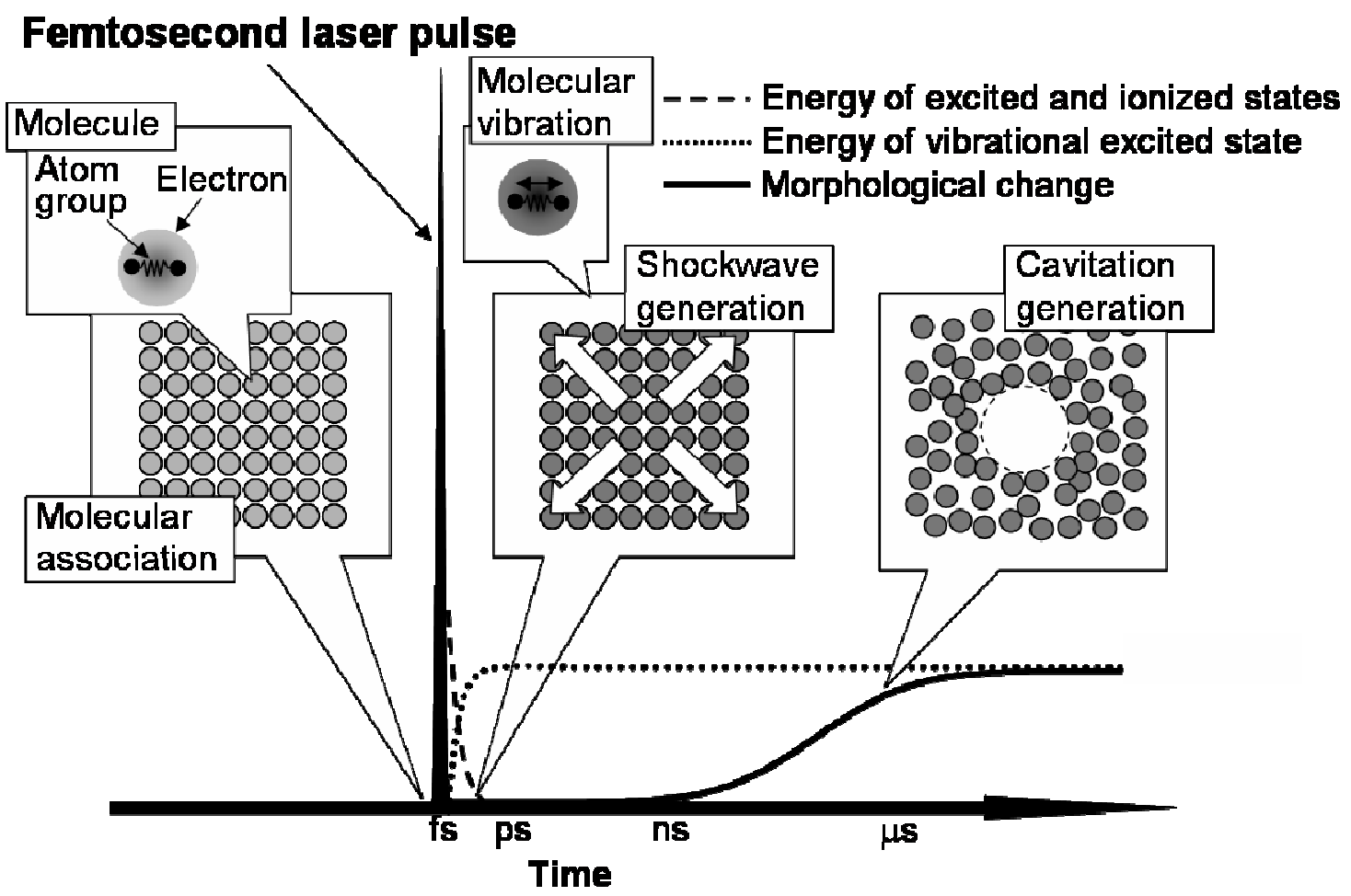

Fig. 2. Schematical illustration of the mechanism of shockwave and cavitation bubble generation

\section{SHOCKWAVE AND CAVITATION BUBBLE GENERATION PROCESSES}

The mechanism of shockwave and cavitation bubble generation has been investigated on the basis of femtosecond laser-induced breakdown [8-10]. More precisely, the mechanism for femtosecond laser irradiation has been detailed on the basis of photomechanical mechanism of femtosecond laser ablation [10, 1215], which should be schematically summarized as shown in Fig. 2. The energy of the femtosecond laser pulse is efficiently deposited in a part of a material, consisting of molecular association, through the multiphoton absorption at the laser focal point. Then, excited and/or ionic states are locally generated, whose energy could be quickly converted to vibrational excited states of molecules composing of number of frequency modes, then to the lattice motion with low frequency modes. Such vigorous molecular motions enhanced in a confined space do not only a temperature increase but also a pressure elevation. In the case of liquid, the excessive pressure will relax as a shockwave. After the shockwave propagation, the pressure at the laser focal point will decrease and induce an explosive vaporization, which results in a cavitation bubble formation [8]. The cavitation bubble soon collapses and forms a jet flow of liquid.

The time-evolution of the shockwave and cavitation bubble generated in water was monitored with a high-speed CMOS camera (Photron, FASTCAM). 

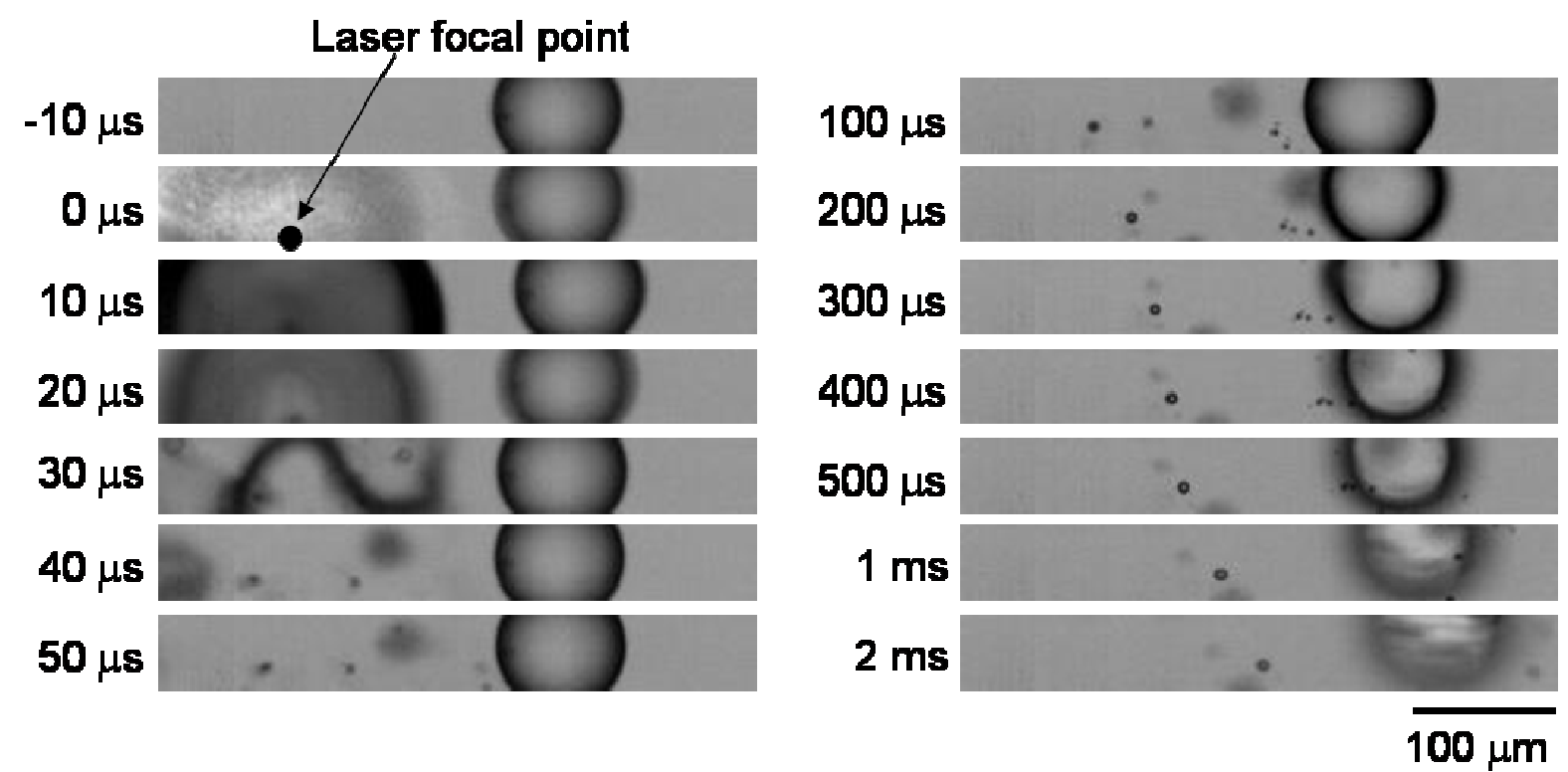

Fig. 3. Time-evolution of shockwave and cavitation bubble monitored by a high-speed imaging device.

Here, instead of NIT3T3 cells described at the inset of Fig. 1, water containing $90 \mu \mathrm{m}$ polystyrene beads was used as a model sample in order to monitor its convection. The transmitted images, whose light source was a halogen lamp equipped on the microscope, were acquired with gate time and frame rate of 166 ns and 100000 frame/sec, respectively. The representative result is shown in Fig. 3 , in which the laser pulse $(24 \mu \mathrm{J} /$ pulse $)$ was focused by a $10 \mathrm{x}$ objective lens (NA 0.45). Immediately after the laser shot $(0 \mu \mathrm{s})$, an emission which will be attributed to plasma emission of water was observed. Simultaneously, the silhouette of the bead was blurred. This means that the bead was accelerated by the impact of the shockwave in the time scale less than $10 \mu \mathrm{s}$. Then a cavitation bubble was generated and collapsed within $30 \mu \mathrm{s}$, where the motion of the bead was synchronized with shape change of cavitation bubble. The collapse of the cavitation bubble initiated a jet flow and small lasting bubbles, by which the bead was further shifted. When the femtosecond laser was focused on the culture medium, similar behaviour of the shockwave and cavitation bubble was observed. Since the size of the bead is in the same order to that of a cell, we can analyze these phenomena to estimate an external force for processing the single cell.

\section{MOTIONS OF SINGLE ANIMAL CELL CULTURED ON A SUBSTRATE}

The behaviour when the femtosecond laser pulse was shot near the NIH3T3 cell in the culture medium was investigated by high-speed imaging. The 


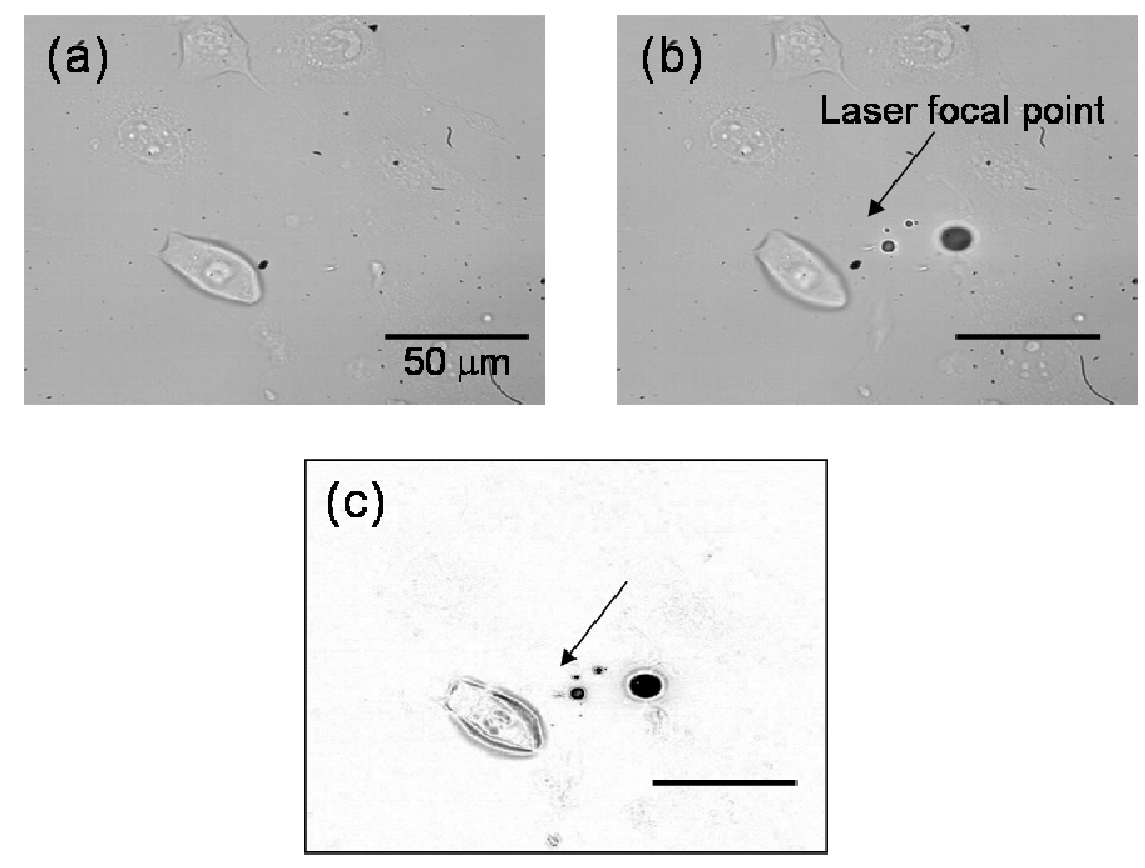

Fig. 4. High-speed microphotographs of NIH3T3 cell before (a) and at $500 \mu$ s (b) after a single-shot femtosecond laser irradiation. Image (c) is difference between (a) and (c).

representative example of transmission images of the NIH3T3 cell is shown in Fig. 4. The laser with $0.8 \mu \mathrm{J} /$ pulse was focused and shot by a $100 \mathrm{x}$ objective lens (NA 1.25) and shot at the position of $20 \mu \mathrm{m}$ away from the cell. In order to see the modification more clearly, a difference image between microphotographs (a) and (b) was calculated as shown in Fig.4 (c). Immediately after the laser irradiation, black spots with size of $1 \sim 25 \mu \mathrm{m}$ were observed at the vicinity of the laser focal point. Simultaneously, the position of the cell was shifted at the opposite side of the laser focal point. Since the shift of the cell is about $5 \mu \mathrm{m}$, it is estimated that the cell was moved with velocity over $10 \mathrm{~mm} / \mathrm{s}$, though the cell adheres tightly on the substrate.

The black spots will be bubbles generated after collapse of the cavitation bubble, so that the cell will be attacked by forces due to shockwave and cavitation bubble generations in this time scale. Namely it is considered that the cell motion is induced by mechanical force. Although there is another possibility that the cell motion is led by cellular biological processes, such as synthesis, polymerization, and depolymerization processes of cytoskeleton, this time scale is too short to initiate them (See section 6). On the other hand, we have also tried to manipulate the NIH3T3 cells using a conventional laser trapping, where optical pressure by near infrared laser was applied, however, it was impossible. This result obviously indicates that the mechanical force due to the shockwave and cavitation bubble is stronger than that of the trapping and will be an effective perturbation enabling manipulation of single cells. 


\section{REGENERATION PROCESS OF CELL MONITORED BY TOTAL INTERNAL REFLECTION FLUORESCENECE IMAGING}

The regeneration process of the cell after the detachment was evaluated by monitoring actin stress fibres connected with the substrate. Actin is a cytoskeleton protein and forms actin filament. Three-dimensional network of actin stress fibre, which is an association of actin filaments, provides mechanical support for the cell, determines the cell shape, and enables cell movements. Here, the actin stress fibre was visualized by binding it with enhanced green fluorescence protein (EGFP). The NIH $3 \mathrm{~T} 3$ cells were transfected with pEGFPactin plasmid (Clontech) and transfectin (Biorad) according to the standard procedures and hatched in a $\mathrm{CO}_{2}$ incubator $\left(5 \% \mathrm{CO}_{2}\right.$ in air) at $37{ }^{\circ} \mathrm{C}$ for 2 days. The actin stress fibres at the interface between the cell and substrate were selectively monitored by total internal reflection fluorescence (TIRF) imaging, whose experimental setup is schematically shown in Fig. 5. A diode pumped solid state (DPSS) laser $(473 \mathrm{~nm})$ led to the edge of a 60x objective lens (NA 1.45) shines up through a glass coverslip at a shallow angle and an evanescent wave probes only an interface layer of the cell adhered on the substrate. Alternatively, the epi-fluorescence image was also acquired by shifting the DPSS laser beam to the centre of the objective lens. These images were acquired by an electron multiplier CCD camera (HAMAMATSU, C9100).

Transmission, epi-fluorescence, and TIRF images before laser irradiation are shown in Figs. 6 (a), (b), and (c), respectively. Although the pEGFP-actin is expressed in the whole cell at the centre of the pictures, only the actin fibres at the interface between the cell and substrate were selectively detected in the TIRF image (c). Hence, the bright lines in TIRF image can be attributed to actin stress fibres which adhered on the substrate. The time evolution of the cell when the laser with $0.3 \mu \mathrm{J} /$ pulse was focused at the ventral side of the cell by the $60 \mathrm{x}$ objective lens is summarized in Figs. 6 (d-f). Immediately after the laser shot,

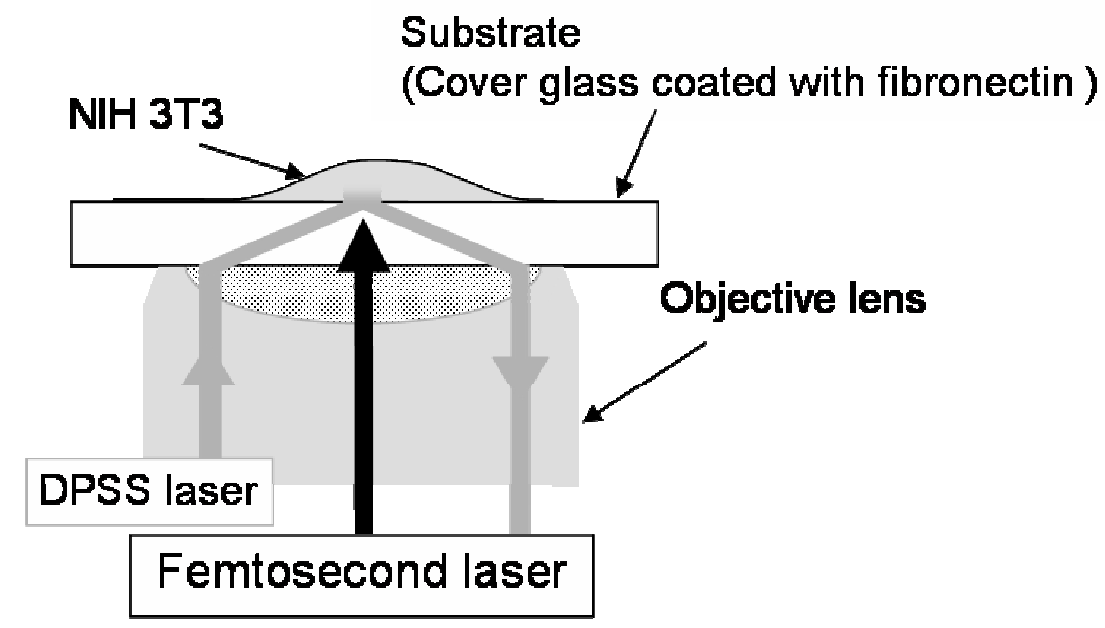

Fig. 5. Schematic illustration of TIRF imaging using high NA objective lens. 

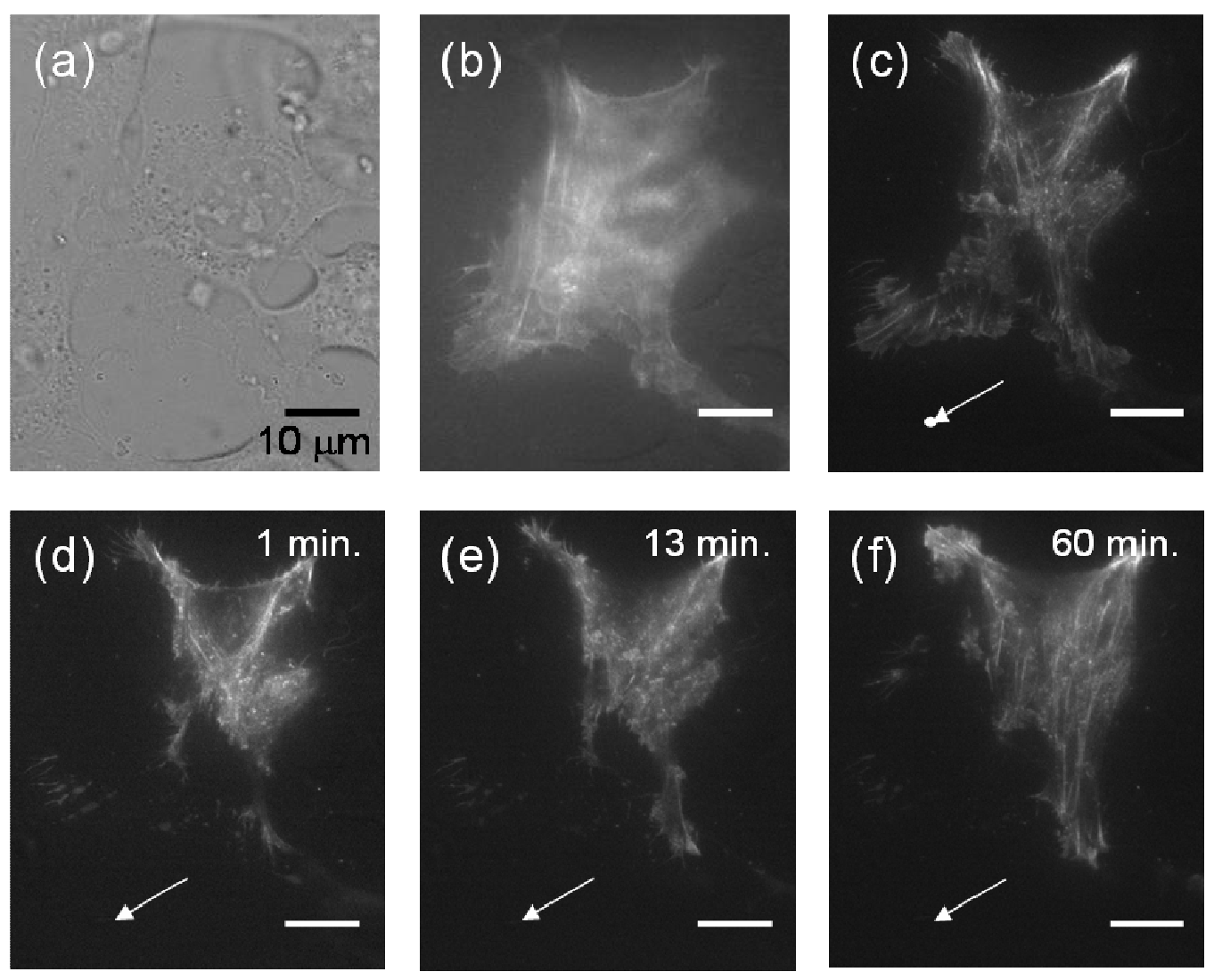

Fig. 6. Transmission (a), Epi-Fluorescence (b), and TIRF (c-f) images of NIH3T3 cell before (a-c) and after (d-f) the femtosecond laser irradiation. Arrows indicate the laser focal point.

fluorescence of actin filaments near the laser focal point were lost. Simultaneously, the averaging intensity of the fluorescent of the actin was increased in the area far from the laser focal point.

The loss of the actin filaments near the laser focal point will be due to the detachment of the cytoskeleton from the culture substrate. The detachment will also induce relaxation of a tension of the cytoskeleton, which is originated in the process of cell adhesion and migration. On the other hand, at the area far from the laser focal point, there is a possibility that the generation of cytoskeleton in the cell is enhanced, because the increase of the fluorescence intensity means that the actin molecules are accumulated at the adhesion area.

At a few tens minutes after the laser irradiation, the actin filament was regenerated from the adhesion area, and the regeneration proceeded as shown in Figs. 6 (e) and (f). Of course, the actual cell viability can not be evaluated by this method, because the cell regeneration strongly depends not only on the laser irradiation but also on the cell culture conditions. For example, even under the normal culture condition, the activity of the cell is prevented when much amount of the pEGFP-actin is expressed. When the pEGFP-actin is not expressed in the cell, about $80 \%$ cells perfectly detached from the culture substrate were adhered again on the culture substrate, which has been already reported [11]. 


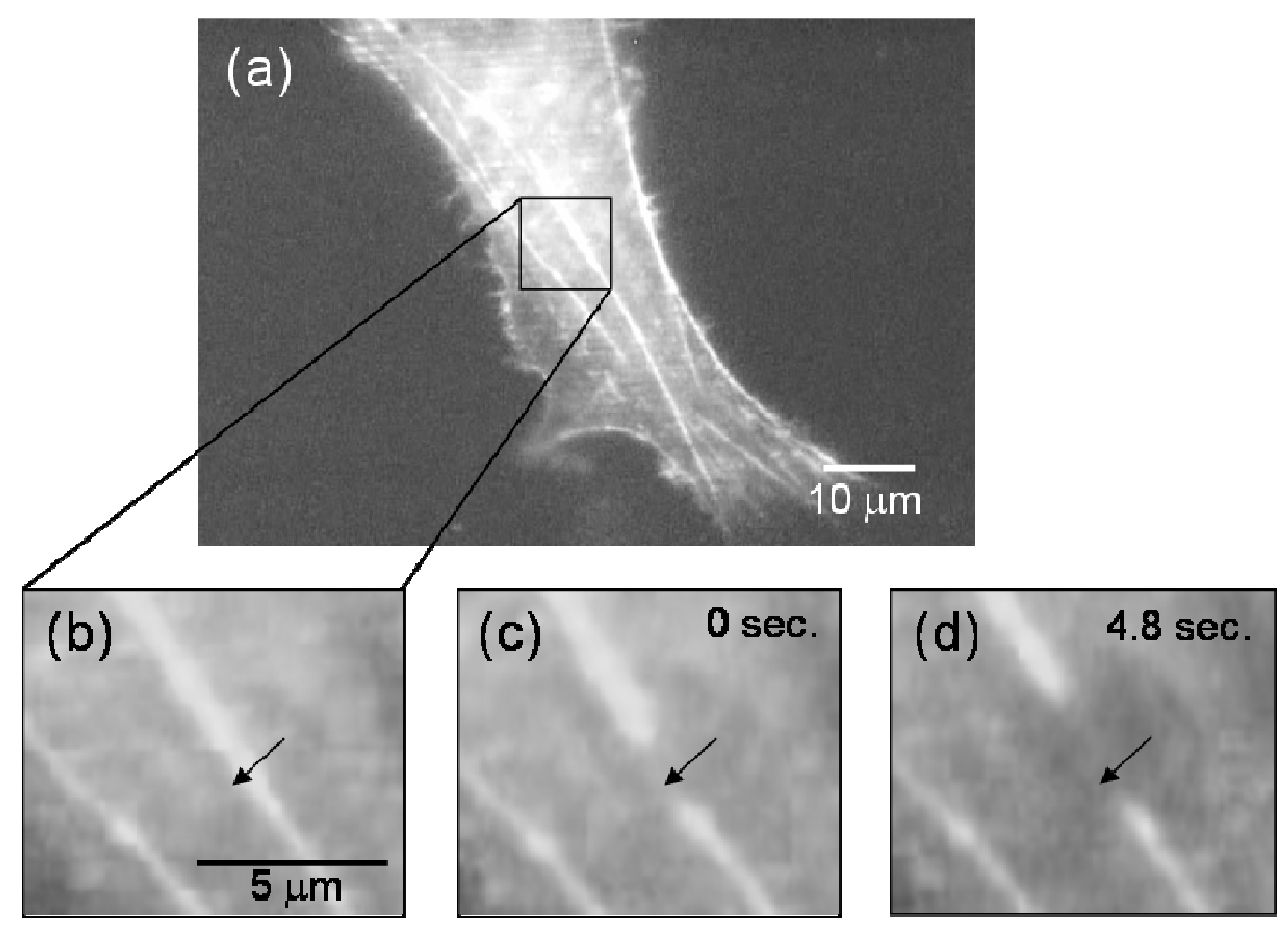

Fig. 7. Fluorescence images of actin stress fibres in NIH3T3 cellafter before $(a, b)$ and after (c, d) a single-shot femtosecond laser irradiation. Arrows indicate laser focal point.

\section{DIRECT CUTTING OF SINGLE ACTIN STRESS FIBRE}

The actin stress fibre was locally cut by direct irradiation of femtosecond laser and the generation process was monitored. In order to obtain fluorescence images, a diode pumped solid state (DPSS) laser $(473 \mathrm{~nm})$ was led to the centre of a 100x objective lens (NA. 1.25). The fluorescence of the pEGFP-actin inside the cell before and after the laser irradiation was imaged by a cooled CCD camera (Roper Scientific, CoolSNAP-HQ).

The fluorescence images when a single-shot femtosecond laser with energy of $4 \mathrm{~nJ}$ was focused on the actin stress fibre are shown in Figs. 7. The focal spot darkened immediately after the laser irradiation (c) and the dark area was increased along the actin stress fibre with time (d). This behaviour will arise from contraction of the actin stress fibre, which is considered to be due to the release of elastic energy of the actin stress fibre. Indeed, Kumar et al, recently reported the same behavior of an actin stress fibre [6]. After the contraction, the fibre was further shrunk in the time scale of a few minutes. In this process, the distance between cut edges was increased linearly. It suggests that an actin depolymerization was initiated in this time scale.

Fig. 8 shows fluorescence images after the relaxation of the cell tension, where the realignment process of the actin stress fibre was observed in a few tens of minutes. Its recovery progressed with the appearance of thin individual microfilaments. It is worth noting that the actin stress fibre did not lose its 

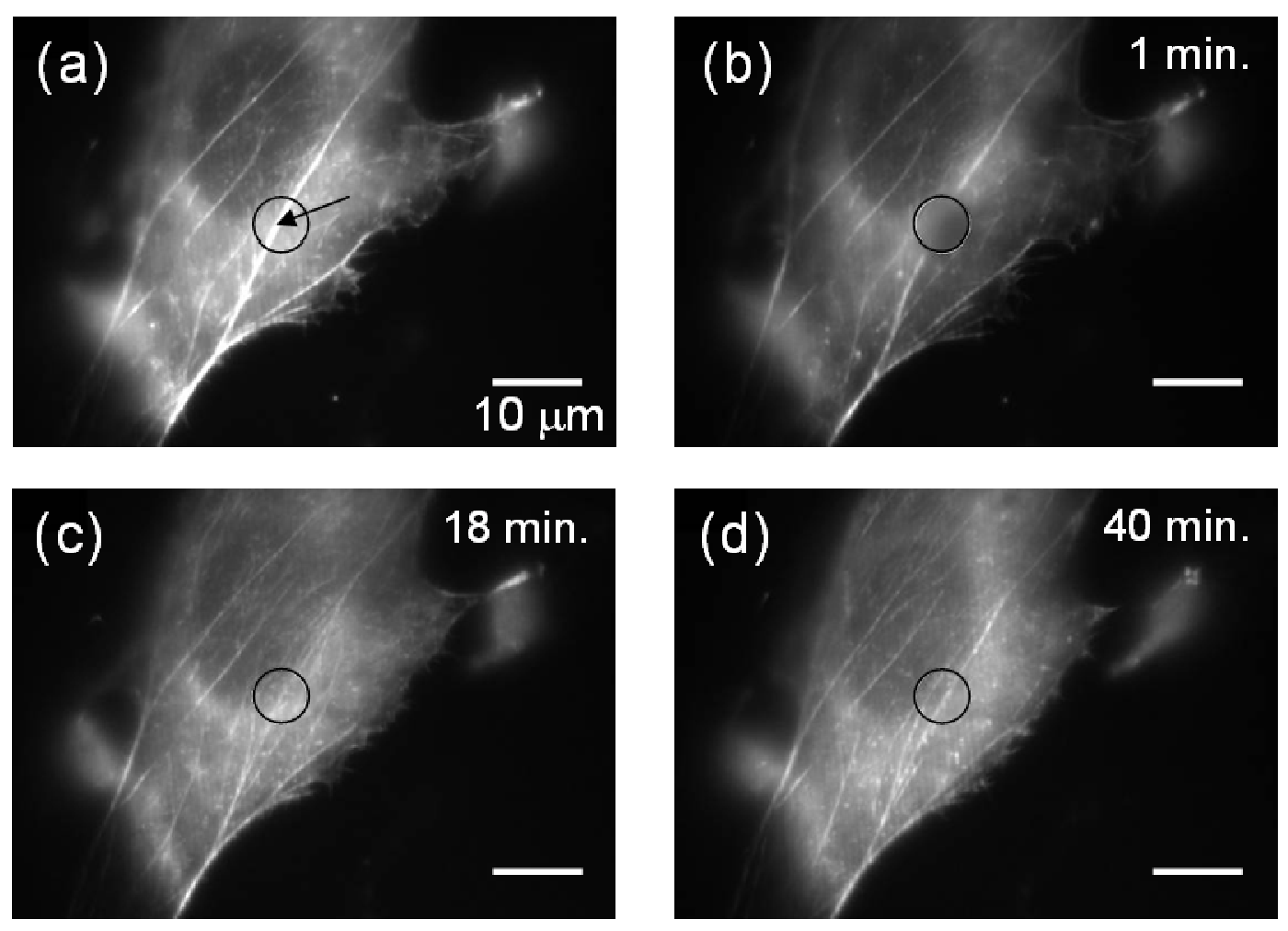

Fig. 8. Fluorescence images of actin stress fibres before (a) and after (b-d) a single-shot laser irradiation. Arrow in circle indicates the laser focal point.

directionality of the elongation even after the femtosecond laser dissection. The realignment process of the actin stress fibres has been also investigated by affecting an external stress to the scaffold, where the actin stress fibres detached from the scaffold was adhered again and realigned in the time scale of a few tens minutes [16]. Since the realignment speed is in good agreement with that after the femtosecond laser dissection, it is suggested that the cell activity was not much changed by the mechanical stress induced by the femtosecond laser irradiation.

\section{CONCLUSION}

In this chapter, we have summarized cellular events when an animal cell is processed by a single-shot focused femtosecond laser. The mechanical influence due to the laser irradiation is not localized at the laser focal point but propagated to the whole of the cell. This result indicates that the focused femtosecond laser has original characteristic as a mechanical perturbation to stimulate the cell. In parallel with the present work, this perturbation of shockwave and cavitation bubble should be applied to develop new methodology for single cell control, such as micro injection of extracellular materials to a single cell, single cell patterning, and so on. 


\section{ACKNOWLEDGEMENTS}

This work was partly supported by CREST of JST (Japan Science and Technology Corporation) and the Grant-in-Aid Project on Scientific Research (KAKENHI) on Priority Area on "Molecular Nano Dynamics" from the MEXT.

\section{REFERENCES}

[1] K. König, Histochem. Cell Biol., 114 (2000) 79.

[2] U. K. Tirlapur and K. König, The Plant J., 31 (2002) 365.

[3] M. F. Yanik, H. Cinar, H. N. Cinar, A. D. Chisholm, Y. Jin and A. Ben-Yakar, Nature, 432 (2004) 882

[4] W. Watanabe, N. Arakawa, S. Matsunaga, T. Higashi, K. Fukui, K. Isobe and K. Itoh, Opt. Express, 12 (2004) 4203.

[5] A. Heisterkamp, I. Z. Maxwell, E. Mazur, J. M. Underwood, J. A. Nickerson, S. Kumar and D. E. Ingber, Opt. Express, 13 (2005) 3690.

[6] S. Kumar, I. Z. Maxwell, A. Heisterkamp, T. R. Polte, T. P. Lele, M. Salanga, E. Mazur and D. E. Ingber, Biophys. J., 90 (2006) 3762.

[7] R. Yasukuni, J. A. Spitz, R. Méallet-Renault, T. Negishi, Y. Hosokawa, T. Asahi, C. Shukunami, Y. Hiraki and H. Masuhara, submitted to Appl. Surf. Sci. (2006).

[8] J. Noack, D. X. Hammer, G. D. Noojin, B. A. Rockwell and A. Vogel., J. Appl. Phys., 83 (1998) 7488.

[9] A. Vogel and V. Venugopalan, Chem. Rev., 103 (2003) 577.

[10] A. Vogel, J. Noack, G. Hüttman and G. Paltauf, Appl. Phys. B, 81 (2005) 1015.

[11] Y. Hosokawa, J. Takabayashi, S. Miura, C. Shukunami, Y. Hiraki and H. Masuhara, Appl. Phys. A, 79 (2004) 795.

[12] Y. Hosokawa, M. Yashiro, T. Asahi and H. Masuhara, J. Photochem. Photobio. A, 142 (2001) 197.

[13] Y. Hosokawa, M. Yashiro, T. Asahi, H. Fukumura and H. Masuhara, Appl. Surf. Sci., 154-155 (2000) 192.

[14] G. Paltauf and P. E. Dyer, Chem. Rev., 103 (2003) 487.

[15] L. V. Zhigilei and B. J. Garrison, J. Appl. Phys., 88 (2000) 1281.

[16] K. Naruse, T.Yamada and M. Sokabe, Am. J. Physiol., 43 (1998) 1532. 